\title{
Simulations of the ILC Electron Gun and Electron Bunching System
}

\author{
Christian Bernt Haakonsen \\ Office of Science, SULI Program \\ McGill University \\ Stanford Linear Accelerator Center \\ Menlo Park, CA
}

August 25, 2006

Prepared in partial fulfillment of the requirements of the Office of Science, U.S. Department of Energy Science Undergraduate Laboratory Internship (SULI) Program under the direction of Axel Brachmann at the Stanford Linear Accelerator Center.

Participant:

Signature

Research Advisor:

Signature 


\section{Table of Contents}

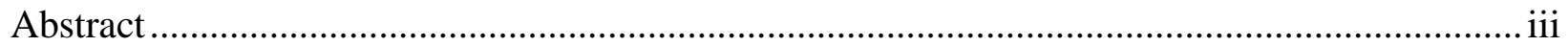

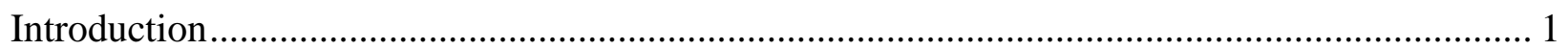

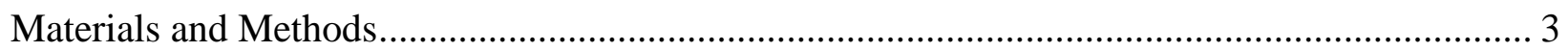

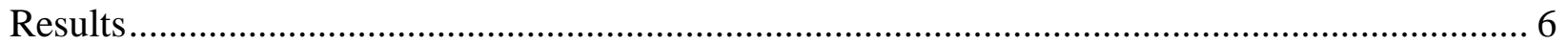

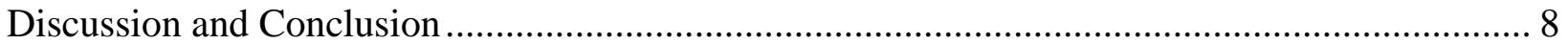

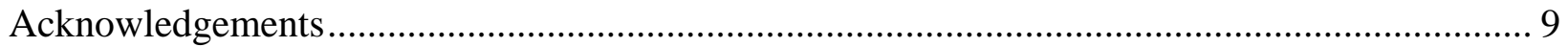

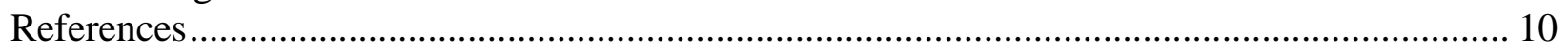

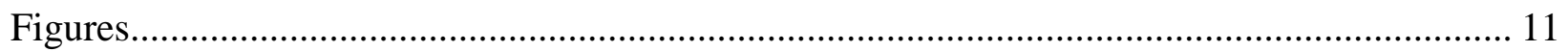

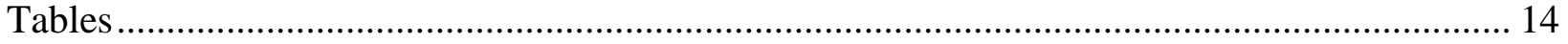




\begin{abstract}
Simulations of the ILC Electron Gun and Electron Bunching System. CHRISTIAN BERNT HAAKONSEN (McGill University, Montreal, Quebec, CANADA) AXEL BRACHMANN (Stanford Linear Accelerator Center, Menlo Park, CA).
\end{abstract}

The International Linear Collider (ILC) is a proposed electron-positron collider, expected to provide insight into important questions in particle physics. A part of the global R\&D effort for the ILC is the design of its electron gun and electron bunching system. The present design of the bunching system has two sub-harmonic bunchers, one operating at $108 \mathrm{MHz}$ and one at 433MHz, and two 5-cell 1.3 GHz (L-band) bunchers. This bunching system has previously been simulated using the Phase and Radial Motion in Electron Linear Accelerators (PARMELA) software, and those simulations indicated that the design provides sufficient bunching and acceleration. Due to the complicated dynamics governing the electrons in the bunching system we decided to verify and expand the PARMELA results using the more recent and independent simulation software General Particle Tracer (GPT). GPT tracks the motion and interactions of a set of macro particles, each of which represent a number of electrons, and provides a variety of analysis capabilities. To provide initial conditions for the macro particles, a method was developed for deriving the initial conditions from detailed simulations of particle trajectories in the electron gun. These simulations were performed using the Egun software. For realistic simulation of the L-band bunching cavities, their electric and magnetic fields were calculated using the Superfish software and imported into GPT. The GPT simulations arrived at similar results to the PARMELA simulations for sub-harmonic bunching. However, using GPT it was impossible to achieve an efficient bunching performance of the first L-band bunching cavity. To 
correct this, the first L-band buncher cell was decoupled from the remaining 4 cells and driven as an independent cavity. Using this modification we attained results similar to the PARMELA simulations. Although the modified bunching system design performed as required, the modifications are technically challenging to implement. Further work is needed to optimize the L-Band buncher design. 


\section{Introduction}

To answer fundamental questions that have arisen in particle physics, physicists are planning the International Linear Collider (ILC). The ILC will be a $1 \mathrm{TeV}$ electron-positron collider, designed to probe the same energy scale as the nearly completed Large Hadron Collider (LHC). Compared to the proton-antiproton collisions of the LHC, the ILC's electron-positron collisions will provide a better signal to noise ratio, no bias towards producing strongly interacting particles, and a well known center of mass frame for each elementary particle collision. This will allow the ILC to study the properties of the particles discovered at the LHC, and discern different theories that predict similar results at the LHC.

A part of the global R\&D effort for the ILC is the design of its electron gun and electron bunching system. These are currently being developed at the Stanford Linear Accelerator Center, and are the focus of this paper. The electron gun generates electrons (3.2 nC per bunch) through the illumination of a photocathode by a 2 ns laser pulse. A cathode bias of $-120 \mathrm{keV}$ accelerates the electrons to $\beta=V / c=0.59$ at the gun exit. Since the electron bunch extends more than a full wavelength of the $1.3 \mathrm{GHz}$ (L-band) driving frequency of the main ILC linac, sub-harmonic bunching is required for the initial shortening of the bunch, before L-band bunchers compress and accelerate to produce a $20 \mathrm{ps}, 10 \mathrm{MeV}$ electron bunch .

The bunching system outlined in [1] for the TESLA accelerator is used as the preliminary ILC bunching design. In this design a first sub-harmonic buncher (SHB1) is operated at 108 $\mathrm{MHz}$, and a second at $433 \mathrm{MHz}$ (SHB2) at a distance of $2.02 \mathrm{~m}$. The first of two L-band bunchers is located $0.33 \mathrm{~m}$ from the second subharmonic buncher, with the second L-band buncher spaced $0.1 \mathrm{~m}$ from the first. Each L-band buncher is a 5-cell RF standing wave cavity, and both share a single klystron. 
Computer simulations are an important tool in testing and improving electron gun and bunching designs. In the electron gun a solution to Poisson's equation is needed to calculate the electron trajectories. Also, Child's law gives the space-charge limited current which cannot be exceeded [2: 46]. Iteratively tracing rays of current and numerically solving Poisson's equation leads to a convergent solution for the currents in the gun. At the exit of the gun it must be evaluated if space-charge effects are significant in the dynamics of the electron bunch. An estimate of the ratio of space-charge to emittance effects on the bunch envelope evolution is given by:

$$
R_{0}=\frac{2 I \sigma^{2}}{I_{0} \gamma^{2} \varepsilon^{2}}
$$

where I is the peak current, $\sigma$ the bunch radius, $\gamma$ the relativistic parameter, $\varepsilon$ the unnormalized emittance, and the characteristic electron current $I_{0}=\frac{e \cdot c}{r_{e}}$ (where $\mathrm{r}_{\mathrm{e}}$ is the radius of an electron) [3]. At $120 \mathrm{keV}$ and the order of magnitude of emittance we expect at the gun exit $\mathrm{R}_{0}$ will be larger than 1, which indicates that space-charge effects must be considered. For a much larger energy or emittance space-charge could be ignored and $R_{0}<<1$. The presence of space-charge effects makes this a many body problem, and it can usually be solved by grouping electrons into macro particles and calculating the motion of the macro particles numerically. If convergence to a solution occurs as the number of macro particles is increased (thus decreasing the number of electrons per macro particle) it is unnecessary to simulate individual electron interactions (which would take prohibitively long on current hardware).

This paper presents the results of simulations of the electron gun and bunching section of the ILC, and compares them to the simulations presented in [1]. Several software tools are used 
in conjunction to simulate the electron bunch propagation as accurately as possible, and areas requiring further study are proposed.

\section{Materials and Methods}

Simulations were preformed on a PC with an Intel Pentium 42.4 GHz CPU and 2 GB of RAM.

The electron gun was simulated using the Egun version 2 (v2) software by entering an r-z cylindrically symmetric gun design, the radius of the emitting area of the cathode, and the cathode bias voltage. In addition the perveance, $\mathrm{K}$, was specified to limit the current to the intended $1.6 \mathrm{~A}$, which in the actual gun will be determined by the laser intensity [4: 36]. Perveance, bias voltage and current are related by:

$$
K=\frac{I}{\sqrt{V^{3}}},
$$

Current and operating voltage result in a perveance of $0.03849 \mu$ perv for this gun. Egun calculates current rays representing the electron trajectories, and calculates each ray's radial position, total current, kinetic energy and angular divergence at the end of the gun. Particle trajectories are used to determine the unnormalized (3) and normalized edge emittance (4) of the beam at the gun exit [2: 57]:

$$
\begin{aligned}
& \varepsilon_{\text {edge }}=4 \cdot \sqrt{\overline{x^{2}} \cdot \overline{x^{\prime 2}}-\overline{x^{2} \cdot x^{\prime 2}}} \\
& \varepsilon_{\text {edge }, N}=\beta \cdot \gamma \cdot \varepsilon_{\text {edge }} .
\end{aligned}
$$

To simulate the bunching system we used the General Particle Tracer v2.71 (GPT) software. Its inputs are a list of fields and elements that act on the electrons, and the initial conditions of the macro particles representing the electrons. The GPT Multiple Run (MR) feature 
allows running the simulation for a several dimensional parameter space. This technique was used to optimize a set of parameters such as field amplitudes, distances between elements, and initial phase of oscillating electromagnetic fields. To increase simulation speed the radial dependence of the axial solenoidal magnetic fields were ignored. This is justified as the electrons propagate near the axis of the solenoidal fields at all times.

The Egun final ray states were used to calculate the initial conditions for the macro particles in GPT. Each ray was taken to represent a number of electrons (GPT input parameter nmacro) corresponding to the ray current. Due to the cylindrical symmetry of the gun, Egun does not give a cylindrical angle in the plane perpendicular to the axis, only a radius. To spread the macro particles from a radial line to a disk we assigned each particle a random angle, $\theta$, in the interval $(0 . .2 \pi)$ to calculate the $\mathrm{x}$ - and $\mathrm{y}$-components of its position. A scaling from $\mathrm{mm}$ to $\mathrm{m}$ was also preformed. The kinetic energy $(T)$, rest mass energy $\left(E_{0}\right)$ and the angular divergence $(\alpha)$ were used with $\theta$ to calculate the following GPT input parameters:

$$
\begin{aligned}
& \gamma \beta_{x}=\left(1+\frac{T}{E_{0}}\right) \sqrt{1-\left(1+\frac{T}{E_{0}}\right)^{-2}} \sin (\alpha) \cos (\theta), \\
& \gamma \beta_{y}=\left(1+\frac{T}{E_{0}}\right) \sqrt{1-\left(1+\frac{T}{E_{0}}\right)^{-2}} \sin (\alpha) \sin (\theta), \\
& \gamma \beta_{z}=\left(1+\frac{T}{E_{0}}\right) \sqrt{1-\left(1+\frac{T}{E_{0}}\right)^{-2}} \cos (\alpha) .
\end{aligned}
$$

These are required by GPT for each macro particle in addition to the three spatial coordinates and number of electrons. The macro particles are started by GPT uniformly as a function of time over 2 ns to closely resemble the actual particle distribution at the gun exit. Because Egun lists 
the rays in order of increasing starting radius at the cathode, the order of the macro particles was randomized before input to GPT to avoid correlation between starting time and initial radius.

To calculate the L-band buncher fields using the Superfish v7 software, two types of cells were considered, central and end cells. The cells are all cylindrically symmetric and specified to Superfish as r-z plane geometry. The central cell is symmetric about its z midpoint, with an RF source on the cell wall at the plane of symmetry. The cell wall was defined as a Dirichlet boundary, whereas the iris planes on both ends were defined as Neumann boundaries. The end cell was extended on one side to account for fringe fields at the end of each cavity. The output from Superfish was an $\mathrm{r}-\mathrm{z}$ grid specifying $\mathrm{E}_{\mathrm{z}}, \mathrm{E}_{\mathrm{r}},|E|$, and $\mathrm{H}$, at each point. The lengths were scaled from cm to m, E components from $\mathrm{MV} / \mathrm{m}$ to $\mathrm{V} / \mathrm{m}$, and $\mathrm{H}$ from $\mathrm{A} / \mathrm{m}$ to $\mathrm{T}$ (factor of $\mu_{0}$ ), before conversion to the GPT data format. To obtain the end cell field at the opposite side of the cavity, the z-coordinates of the original end cell field map were reversed, and the sign of the $\mathrm{E}_{\mathrm{r}}$ components changed to preserve right-handedness.

In addition to the built in functions of 'gdfa', GPT's analysis tool, three custom functions were written to analyze the simulation output. The first was zdist, which calculates the distance between the first and last electron in the bunch. The second was tlen, which calculates the time between the first and last electron passing a point in space. The final custom function was nelectrons, which counts the number of electrons remaining in the bunch, thereby accounting for lost particles. 


\section{Results}

Egun simulations were performed to calculate the emittance as function of laser spot radius on the cathode (Fig. 1). A global minimum was found for a laser radius of $3.6 \mathrm{~mm}$, and local minima for $2.5 \mathrm{~mm}$ and $4.6 \mathrm{~mm}$. The data points below a radius of $2 \mathrm{~mm}$ are inconsistent with Child's law, as too much current is being forced from a small area, and are therefore not further considered. To investigate detail of the three local minima the simulations were repeated while forcing a current of $2.4 \mathrm{~A}$, and the local minima were found to be at the same radii as for 1.6 A. It was found that the positions of the local minima are independent of current, and that they modulate a large overall $r$ dependence, which is current dependent. The gun geometry with equipotential lines and current rays for a laser radius of $3.6 \mathrm{~mm}$ is shown in Fig. 2.

A combination of parameters that would replicate the bunching performance achieved in [1] could not be found. Simulations of a single 5 cell L-band buncher using various input bunch lengths, field strengths and initial phases, failed to produce a single scenario where bunching and acceleration occurred. To achieve the desired bunching and acceleration the phase of the first cell of the first buncher had to be decoupled from the remaining four cells. This is not physically possible without driving the decoupled cell separately from the others, so the second cell of the buncher was changed to an end cell to create a 4-cell buncher that could be separated as a whole from the first single L-band cavity. The fields of the first cell were modified to make a standalone cell by mirroring the field at the center plane of the cavity and changing the sign of the $\mathrm{E}_{\mathrm{r}}$ component on the mirrored side to preserve right-handedness. Thus a cell with fringe fields on both sides was produced. The axial solenoidal field was also strengthened to various degrees to 
improve electron bunch containment. With these alterations the L-band bunchers were made to function properly.

To be able to propagate the laser beam perpendicular to the photocathode plane, a bending magnet was introduced before the first sub-harmonic buncher. The angle was chosen to be $45^{\circ}$, and the recently available GPT element sectormagnet was downloaded and installed to be used as a bending magnet. To improve electron containment by the solenoidal field between the gun and the bending magnet the distance was increased from $0.75 \mathrm{~m}$ to $1.4 \mathrm{~m}$. Furthermore, the single artificial solenoidal field was replaced by a Superfish simulation of two Helmholtz coils with one focusing solenoid before and two focusing solenoids after it. The axial magnetic field before the bending magnet is shown in Fig. 3, and the field after the bending magnet (generated using GPT solenoid elements) is shown in Fig. 4. The z-axis in Fig. 3 is located at an angle of $45^{\circ}$ with respect to the z-axis in Fig. 4.

Table 1 summarizes what was found to be the optimum operating parameters for the bunching system. For these parameters a bunch length of $\sim 20$ ps (FWHM) was observed, with a particle loss of a few percent. A particle was considered lost when its distance from the average z-position exceeded five standard deviations. The final bunch length was found to be very sensitive to the phase of the single L-band cell.

The beam waist through the length of the bunching section is shown in Fig. 5. The full bunch length (zdist) and standard deviation of z (stdz) along the same length is shown in Fig. 6. In Fig. 5 and 6 the particle distribution is plotted with respect to the z-axis. The bending magnet is located at $z=0$. The section $z<0$ is the projection onto the $z$-axis without consideration of the bending angle, causing a $\frac{1}{\sqrt{2}}$ scaling of the z-coordinates. 


\section{Discussion and Conclusion}

By reduction of the laser spot radius from $10 \mathrm{~mm}$ to $3.6 \mathrm{~mm}$ the edge emittance at the end of the gun was reduced by more than an order of magnitude. However, the beam envelope is strongly influenced by space-charge effects at the gun exit ( $R_{0}=4.6$; formula (1)). It may be

possible to find a laser radius that will result in lower collimation at the end of the gun, yet provided a better contained beam waist leading up to the bending magnet due to reduced spacecharge effects. An optimized laser radius at the cathode can provide a possibility for improved bunch propagation compared to the one used in this study. Further, optimized electron gun parameters may also reduce the necessary solenoidal field for beam containment.

The L-band buncher cavities are standing wave structures designed for particles with $\beta \approx 1$. For such a design the field from one cell to another advances one half period during the time it takes particles to move one half wavelength of the wave (one cell length), and thus the particles arrive at the same phase of the wave each time it reaches maximum amplitude. This is not the case for the low energy particles generated by a $120 \mathrm{kV}$ electron gun $(\beta=0.59)$. The field in the first cell will reach a maximum before the particles have traveled a quarter wavelength.

The simulations in this study showed it is impossible to bunch a non-relativistic beam using a $\beta=1$ structure without significant particle losses. The energy transfer of such a system will lead to acceleration and decceleration of leading and trailing parts of the bunch resulting in increased energy spread without a bunching effect. To achieve bunch compression, it was necessary to decouple the phase of the first cell to ensure overlap of bunch arrival time and appropriate phase of the following L-band structure. However, for a realistic bunching system phase decoupling is impossible without spacing the first cell from the remaining four. Therefore, 
the first L-band buncher cell was separated and operated as an independent bunching cavity. The bunch propagation was found to be extremely sensitive to the initial phase of the single L-band cell compared to the other RF structures of the system. This may be problematic for a real design. Improvements of the present solution are possible. In particular a low $\beta$ L-band bunching structure should be considered.

The Parmela simulations outlined in [1] using a $\beta=1$ L-band buncher should be further reviewed to analyze details of this design. We emphasize that the fields of standing wave cavities used in the GPT simulation are generated by a Superfish model and accounts for the radial electric field components, whereas the previous PARMELA simulations used a simplified model with only axial fields. This may be a contribution to the different performance of the bunching system in the GPT and PARMELA simulations.

\section{Acknowledgements}

The research described took place at the Stanford Linear Accelerator Center, and I would like to thank my mentor, Axel Brachmann, for patient guidance as I familiarized myself with a new subject area, and through the process of producing a scientific paper. I would also like to thank Adam Edwards for several early edits and advice on improving my scientific writing.

The United States Department of Energy, Office of Science has my gratitude for organizing and funding the SULI program, without which this work would not have been possible. 


\section{References}

[1] A. Curtoni and M. Jablonca; Study of the TESLA preaccelerator for the polarised electron beam. TESLA 2001-22(2)

[2] M. Reisner, Theory and Design of Charged Particle Beams. New York: John Wiley and Sons, Inc., 1994

[3] S.G Anderson, et al., "Space-charge effects in high brightness electron beam emittance measurements," Physical Review Special Topics - Accelerators and Beams., vol. 5, 014201, Jan. 2002

[4] W. B. Herremannsfeldt, Egun - an Electron Optics and Gun Design Program. Stanford Linear Accelerator Center, Stanford University, Stanford, CA. SLAC - 331, Oct. 1988 


\section{Figures}

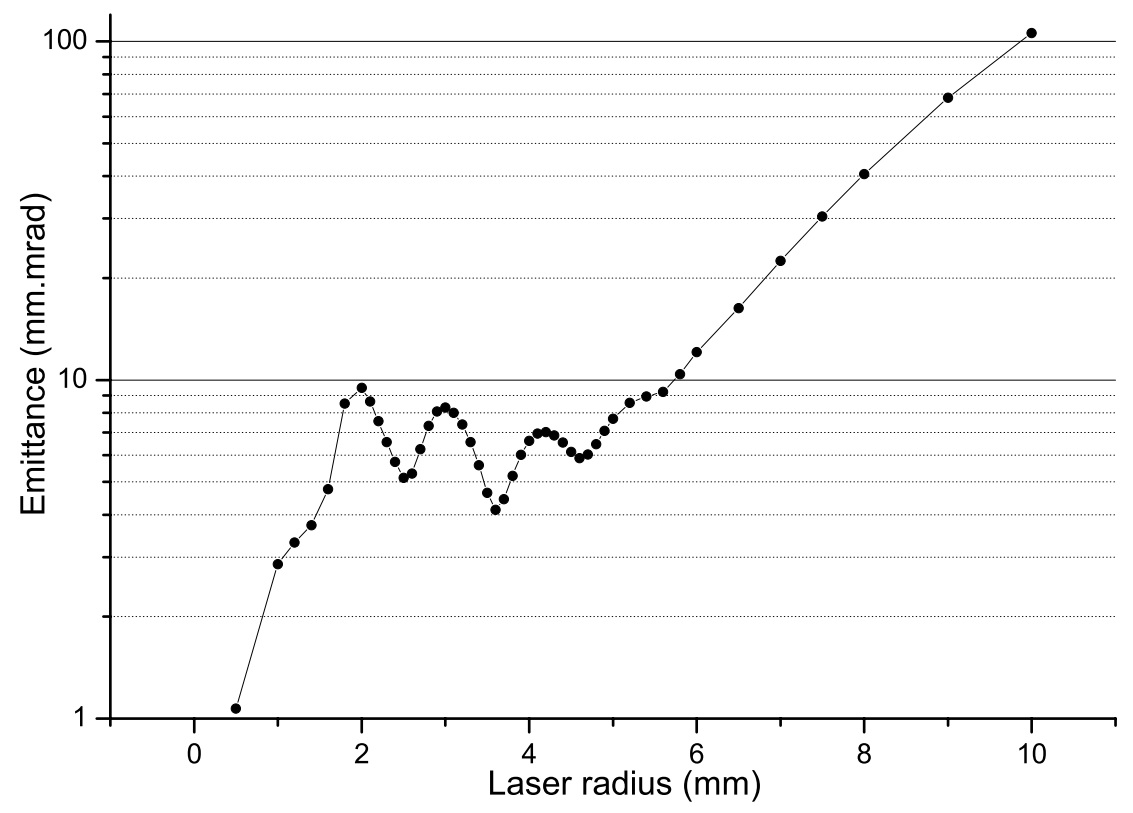

Fig. 1: Emittance of current rays at end of gun as a function of laser spot radius at photocathode

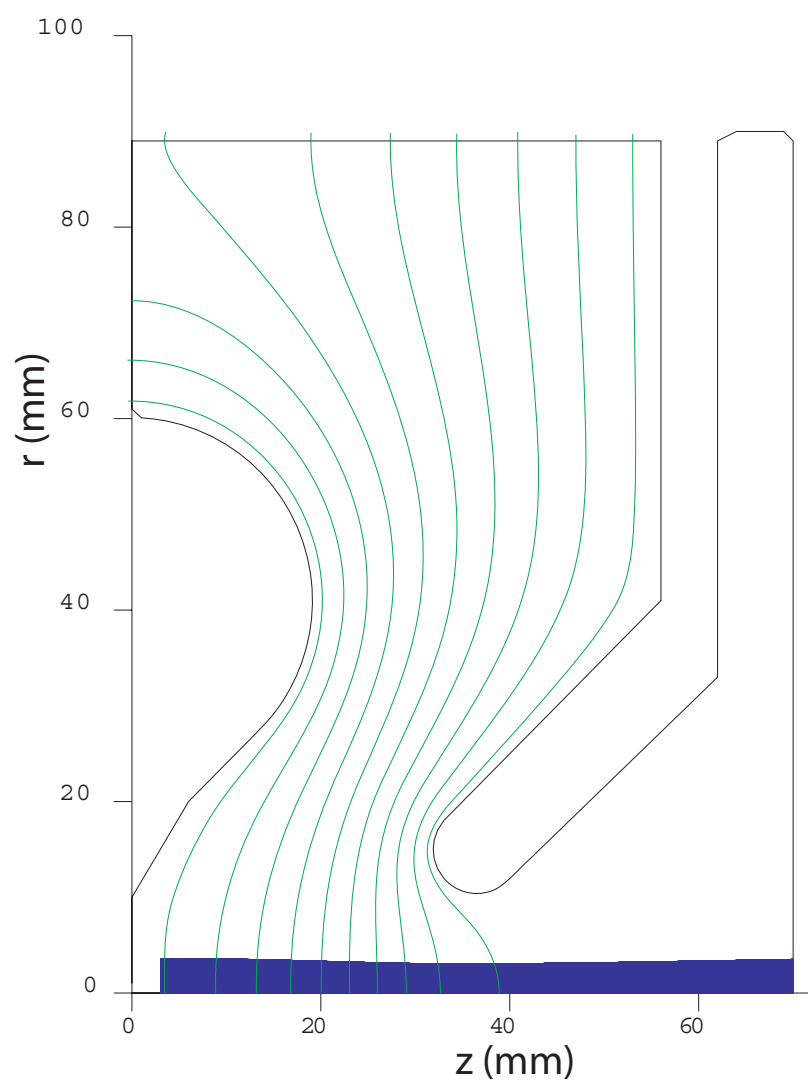

Fig. 2: Equipotential lines (green), geometry (black) and current rays (blue) in electron gun 


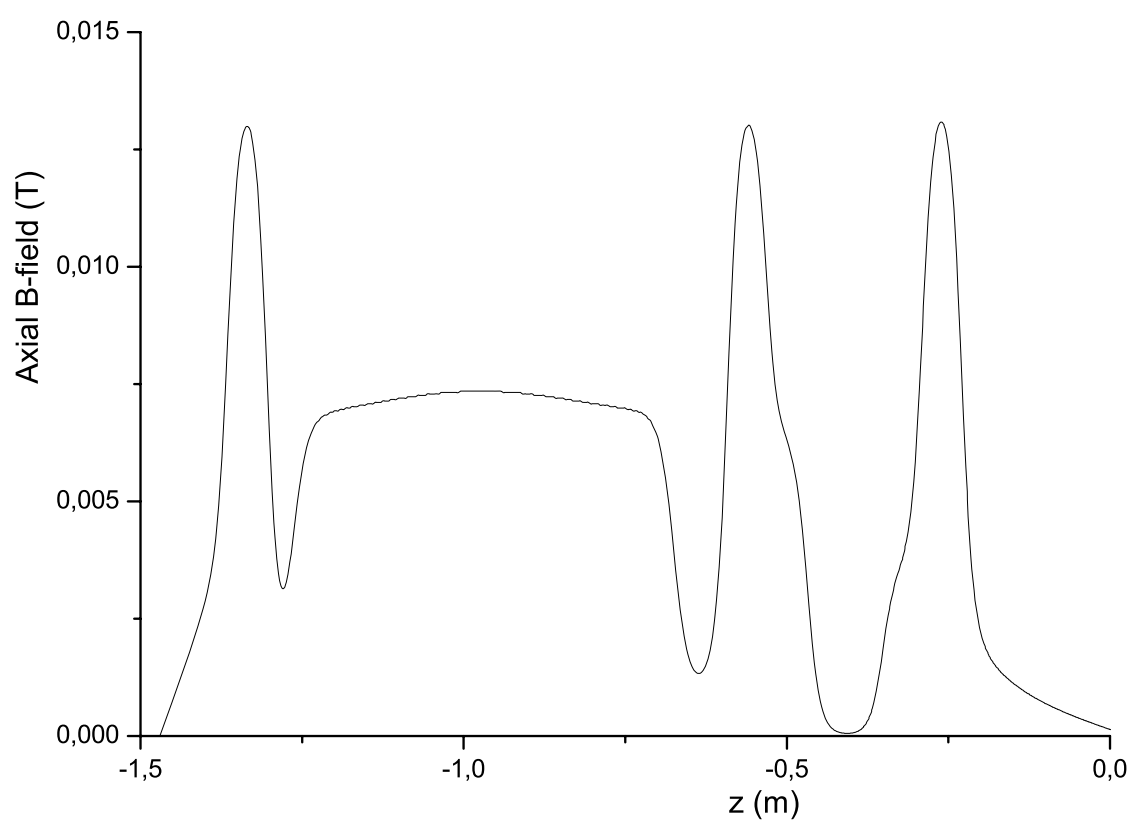

Fig. 3: Axial magnetic field before bending magnet

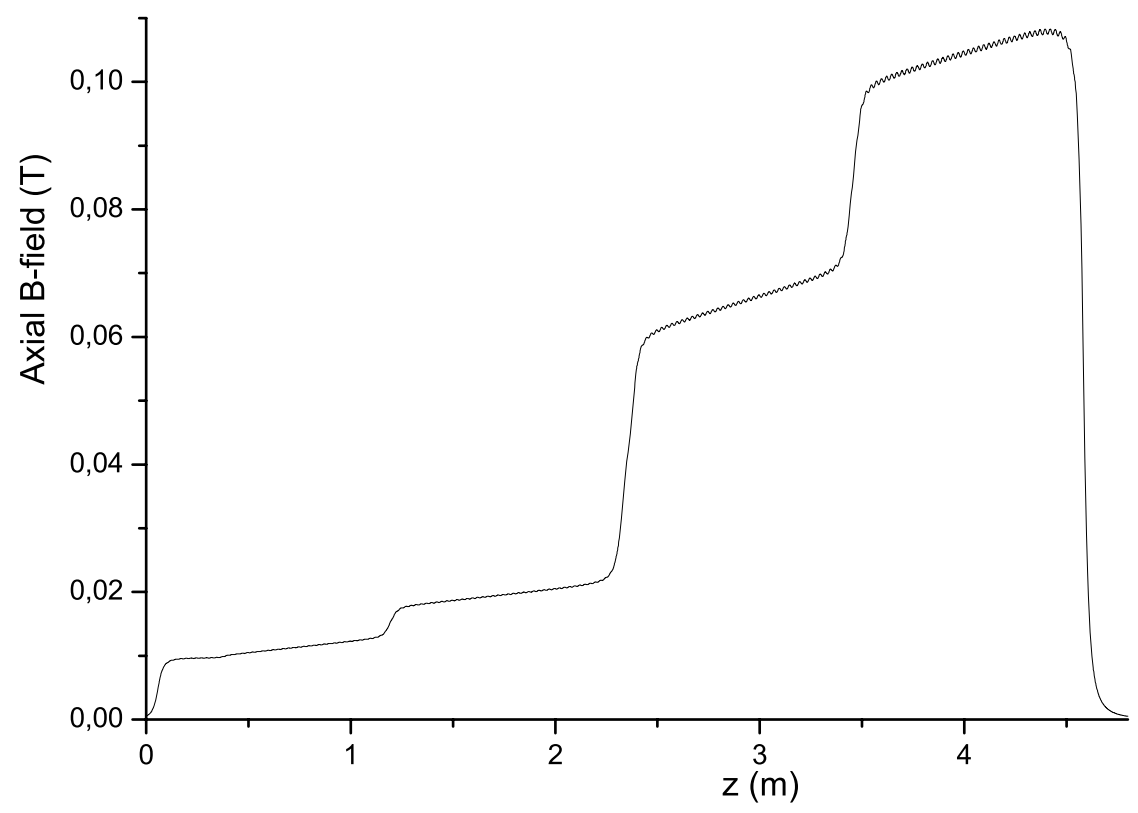

Fig. 4: Axial magnetic field after bending magnet 


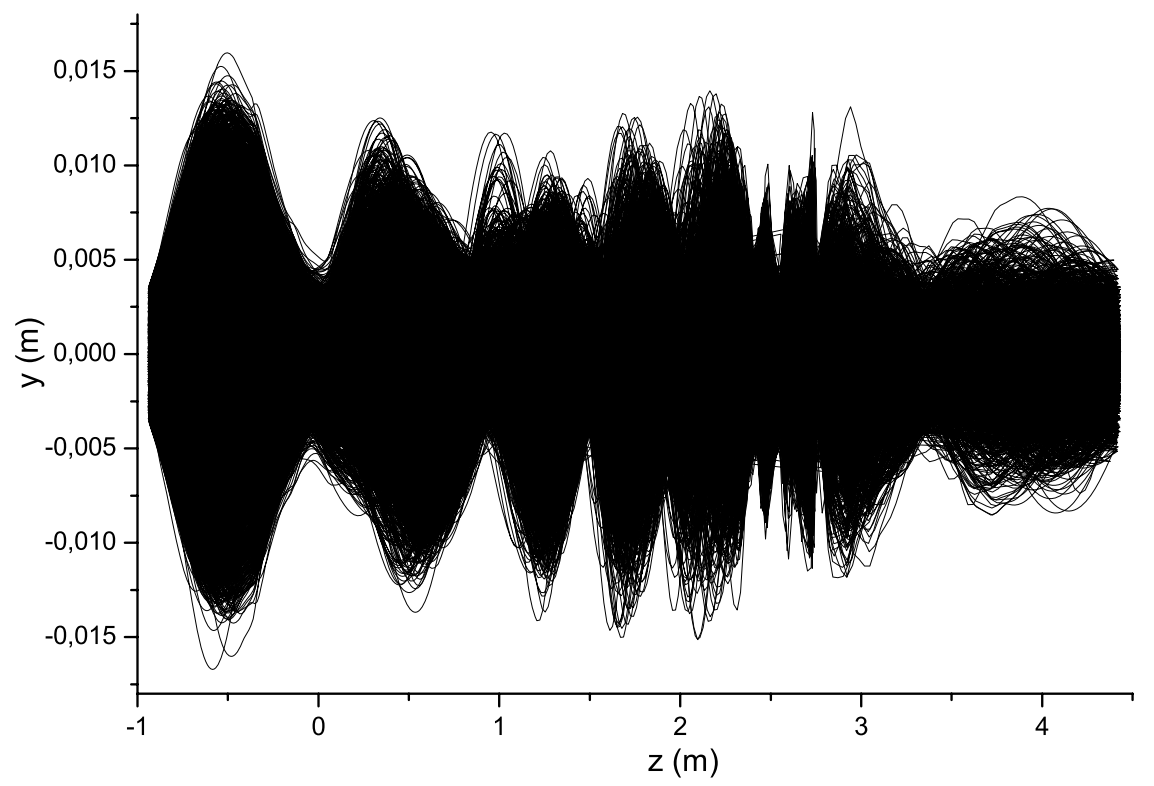

Fig. 5: Macro particle trajectories (in y-z plane) from gun exit through bunching section

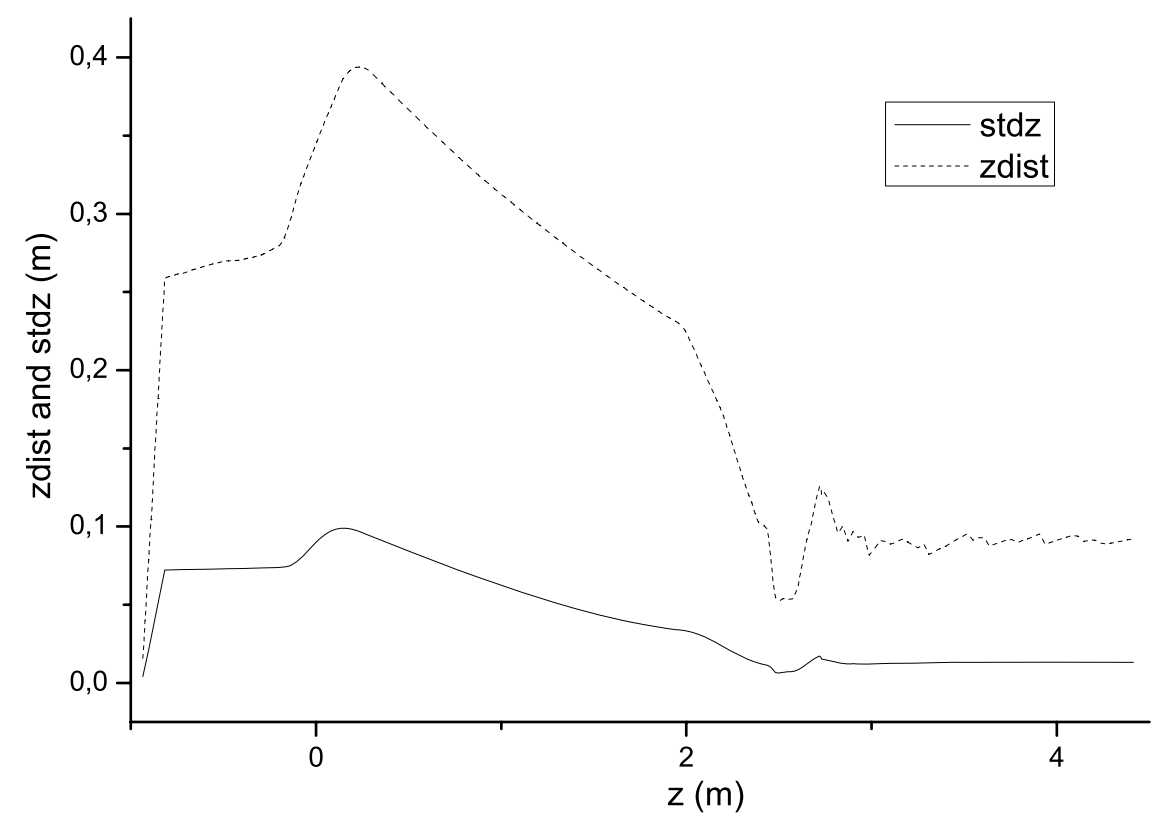

Fig. 6: Maximum axial particle distance (zdist) and standard deviation of z-coordinates (stdz) 


\section{Tables}

\begin{tabular}{|l|c|c|c|c|c|}
\hline Parameter & SHB1 & SHB2 & L-Band cell & $\begin{array}{c}4 \text { cell L-band } \\
\text { Buncher }\end{array}$ & $\begin{array}{c}5 \text { cell L-band } \\
\text { Buncher }\end{array}$ \\
\hline Frequency [MHz] & 108 & 433 & 1300 & 1300 & 1300 \\
\hline Phase angle $\left.{ }^{\circ}{ }^{\circ}\right]$ & 100 & 120 & 216 & 190 & 170 \\
\hline Voltage $[\mathrm{kV}]$ & 65 & 70 & & & 11.4 \\
\hline Field strength $\mathrm{E}_{\mathrm{z}}[\mathrm{MV} / \mathrm{m}]$ & & & 12.3 & 11.4 & \\
\hline
\end{tabular}

Table 1: Optimum operating parameters for the bunching system 